\title{
Inversão dos Dados de Medidas das Resistividades do Solo
}

\author{
Celsa Herminia de Melo Maranhão, Valcir João da Cunha Farias, \\ Instituto de Ciências Exatas e Naturais, UFPA \\ UFPa, Rua Augusto Correa, 01-66075-110, Belém, Pa \\ E-mail: celsa@ufpa.br, Valcir@ufpa.br
}

Brígida R. P. da Rocha

Instituto de Tecnologia, UFPA

UFPa, Rua Augusto Correa, 01-66075-110, Belém-Pa

E-mail: brigida@ufpa.br

Resumo: No estudo de dados de polarização induzida, a aplicação do método de GaussNewton para o processo de inversão aliado com a aplicação do método dos elementos finitos para a modelagem direta mostrou-se eficiente, porém com um alto custo computacional. Aplicando os mesmos métodos para o estudo da resistividade elétrica do solo, os resultados obtidos tão bons quanto em dados de polarização induzida e com menor custo computacional.

\section{I - Introdução}

A modelagem direta e inversa de dados de resistividade elétrica do solo vem sendo amplamente estudada nos últimos anos $[1,2,3,4,6,8]$. O principal propósito da sondagem geoelétrica é obter a resistividade aparente do subsolo considerando o arranjo de eletrodo usado e a estrutura geológica investigada.

A modelagem inversa busca, a partir de informações de resistividade aparente do solo, determinar a distribuição de resistividade do subsolo, ou seja, pode-se criar uma imagem tomográfica da distribuição da resistividade do solo. A interpretação qualitativa e quantitativa de sondagem geoelétrica requer algoritmos eficientes e rápidos de modelagens direta e inversa.

Maranhão et al [2] desenvolveram um algoritimo para modelagem direta de dados de resistividade para a aplicação em aterramento elétrico. Farias et al [4]. Aplicou o método dos elementos finitos para a modelagem direta de dados de polarização induzida para meios 2D e 3D seu algorítimo apresentou um bom desempenho.

Farias et al [3] desenvolveu um processo de inversão de dados de polarização induzida utilizando o método de Gauss-Newton, tal procedimento de mostrou eficiente, porém o custo computacional foi elevado. Como em polarização induzida os valores de resistividades são números complexos, acredita-se que em dados de resistividade elétrica do solo o procedimento de [3] seja também eficiente, porém com um menor custo computacional que para os dados de polarização induzida. O objetivo deste trabalho é aplicar o método de Gauss-Newton para realizar a inversão de dados de resistividade elétrica do solo.

\section{Modelagem direta}

Na modelagem direta foi aplicado o método dos elementos finitos na equação:

$$
-\nabla \cdot(\sigma(x, z) \nabla \tilde{V}(x, \lambda, z))+\lambda^{2} \sigma(x, z) \tilde{V}(x, \lambda, z)=I \delta\left(x-x_{s}\right) \delta\left(z-z_{s}\right) .
$$

Para maiores detalhes ver [6]. 


\section{Modelagem inversa}

A escolha da função objetivo (ou função custo) é fundamental para o bom desenvolvimento do processo. Assim, a inversão de dados da resistividade foi realizada para minimização da função objetivo quadrática, $C(\bar{x})$, da forma descrita por [8], isto é:

$$
C(\bar{x})=\frac{1}{2}\left\{\mu\left[\left\|\bar{W}_{d} \cdot \bar{e}(\bar{x})\right\|^{2}-\chi_{d}\right]+\left\|\bar{W}_{x}\left(\bar{x}-\bar{x}_{r}\right)\right\|^{2}\right\} .
$$

O fator escalar $\mu$ é o multiplicador de Lagrange (também conhecido como parâmetro de regularização) é um parâmetro que determina a importância dos dois termos da função objetivo; Onde:

$\bar{x} \quad$ é um vetor contendo os parâmetros do modelo (resistividade ou condutividade) de cada subdomínio;

$\bar{x}_{r} \quad$ é um vetor de referencia, contendo informação a priori, tais informações obtidas a partir de testemunhos de rochas;

$\chi_{d} \quad$ é um valor preestabelecido de ajuste dos dados;

Na função objetivo definida pela expressa (2) o segundo termo é denominado de termo de regularização [3]. Esse termo de regularização é importante para introduzir informações a priori, servindo, desta forma, para atenuar as ambiguidades (não-unicidade) da solução do problema inverso e, reduzir possíveis amplificações de erros na determinação dos parâmetros do modelo (resistividade ou condutividade), os quais estão presentes nas medidas devido ao inevitável ruído.

\section{Método de Gauss-Newton}

Para resolver o problema de otimização não-linear emprega-se o método iterativo de Gauss-Newton que é baseado em um modelo quadrático da função custo. Este modelo quadrático é formado levando-se os três primeiros termos da expansão por series de Taylor da função custo (1) em torno da k-ésima iteração $\left(\bar{x}_{k}\right)$, como segue:

$$
C\left(\bar{x}_{k}+\Delta \bar{x}_{k}\right) \approx C\left(\bar{x}_{k}\right)+\bar{g}^{T}\left(\bar{x}_{k}\right) \cdot \Delta \bar{x}_{k}+\frac{1}{2} \Delta x_{k}^{T} \cdot \overline{\bar{G}}\left(\bar{x}_{k}\right) \cdot \Delta \bar{x}_{k} \cdot
$$

Onde $T$ indica transposição conjugada e $\Delta \bar{x}_{k}=\bar{x}_{k+1}-\bar{x}_{k}$ é o incremento do vetor dos parâmetros $\bar{x}_{k}$ em direção ao ponto estacionário da função custo $C(\bar{x}) . \quad \bar{g}(\bar{x})=\nabla C(\bar{x})$ é o vetor gradiente da função custo e é determinado pela expressão seguinte:

$$
\begin{aligned}
\bar{g}(\bar{x})=\nabla C(\bar{x}) & =\left[g_{n} \equiv \frac{\partial C}{\partial x_{n}}, n=1,2,3, \ldots ., N\right] \\
& =\mu \overline{\bar{J}}^{T}(\bar{x}) \cdot \overline{\bar{W}}_{d}^{T} \cdot \overline{\bar{W}}_{d} \cdot \bar{e}(\bar{x})+\overline{\bar{W}}_{x}^{T} \cdot \overline{\bar{W}}_{x} \cdot\left(\bar{x}-\bar{x}_{p}\right),
\end{aligned}
$$

Onde:

$x_{n}$ é a n-ésima componente do vetor dos parâmetros do modelo $\bar{x}$; 
$\overline{\bar{W}}_{x}^{T} \overline{\bar{W}}_{x} \quad$ é a matriz inversa da matriz covariância do modelo a qual representa o grau de confiança no vetor de referencia $\left(\bar{x}_{r}\right)$, é também uma informação a priori para a solução do problema de inversão;

$\overline{\bar{W}}_{d}^{T} \cdot \overline{\bar{W}}_{d} \quad$ é a inversa da matriz covariância dos dados, cujo termo no processo de inversão descreve estimativas das incertezas oriundas de contaminação por ruídos dos dados medidos. De acordo com as estimativas de variância para cada medida, $\overline{\bar{W}}_{d}^{T} \cdot \overline{\bar{W}}_{d}$ estima a correlação entre os erros. Segundo Habashy et al [8] se os dados de medidas são contaminados por um ruído estacionário e descorrelacionado, então $\overline{\bar{W}}_{d}=\operatorname{diag}\left(\delta_{j}\right)$, sendo $\delta_{j}$ o desvio padrão $r m s$ do ruído para a $j$-esima medida;

e $\overline{\bar{J}}(\bar{x})$ é uma matriz cuja dimensão $M \times N$ denominada de matriz Jacobiano sensitividade dado por:

$$
\overline{\bar{J}}(\bar{x})=\left[J_{m n} \equiv \frac{\partial e_{m}}{\partial x_{n}}, m=1,2,3, \ldots \ldots, M ; n=1,2,3, \ldots ., N\right]
$$

e $\overline{\bar{G}}(\bar{x})=\nabla \nabla C(\bar{x})$ é o Hessiano da função custo $C(\bar{x})$ o qual é uma matriz simétrica de ordem $N \times N$ dada por:

$$
\begin{aligned}
\overline{\bar{G}}(\bar{x}) & =\nabla \nabla C(\bar{x})=\left[G_{n m}=\frac{\partial^{2} C}{\partial x_{n} \partial x_{m}}, n, m=1,2,3, \cdots, N\right]= \\
& =\overline{\bar{W}}_{x} \cdot \overline{\bar{W}}_{x}^{T}+\mu\left[\overline{\bar{J}}(\bar{x}) \cdot \overline{\bar{W}}_{d}^{T} \cdot \overline{\bar{W}}_{d} \cdot \overline{\bar{J}}(\bar{x})+\overline{\bar{Q}}(\bar{x})\right],
\end{aligned}
$$

Onde $\overline{\bar{Q}}(\bar{x})=\sum_{m=1}^{M} f_{m}(\bar{x}) F_{m}{ }^{H}(\bar{x})$ e $f_{m}(\bar{x})$ é o m-ésimo elemento do vetor $\bar{f}(\bar{x})=\overline{\bar{W}}_{d} \cdot \bar{e}(\bar{x})$, e

$$
\overline{\bar{F}}_{m}(\bar{x})=\nabla \nabla f_{m}(\bar{x})=\left[\partial^{2} f_{m} / \partial x_{i} \partial x_{j}, i, j=1,2,3, \ldots, N\right]
$$

Note que o método de Gauss-Newton despreza as derivadas de segunda ordem da função custo em relação aos componentes do vetor dos parâmetros do modelo $(\bar{x})$, ou seja, não considera-se o termo $\overline{\bar{Q}}(\bar{x})$ no Hessiano. Assim, o Hessiano, no método de Gauss-Newton, será dado por:

$$
\overline{\bar{G}}(\bar{x})=\overline{\bar{W}}_{x}^{T} \overline{\bar{W}}_{x}+\mu\left[\overline{\bar{J}}^{T}(\bar{x}) \cdot \overline{\bar{W}}_{d}^{T} \cdot \overline{\bar{W}}_{d} \cdot \overline{\bar{J}}(\bar{x})\right]
$$

O mínimo de (2) é obtido quando $\Delta x_{k}$ for um mínimo da função quadrática. 
$\phi(\Delta \bar{x})=\bar{g}^{T}\left(\bar{x}_{k}\right) \cdot \Delta \bar{x}+\frac{1}{2} \Delta \bar{x}^{T} \cdot \overline{\bar{G}}\left(\bar{x}_{k}\right) \cdot \Delta \bar{x}$.

A função $\phi(\Delta \bar{x})$ tem um ponto estacionário (ponto critico) em $\Delta \bar{x}_{0}$, somente se o gradiente de $\phi(\Delta \bar{x})$ for para zero em $\Delta \bar{x}_{0}$, isto é:

$\nabla \phi\left(\Delta \bar{x}_{0}\right)=\bar{g}^{T}\left(\bar{x}_{k}\right)+\overline{\bar{G}}\left(\bar{x}_{k}\right) \cdot \Delta \bar{x}_{0}=0$.

\section{Resultados e Discussão.}

O resultado apresentado é referente a um modelo geológico sintético e para dar maior confiabilidade ao procedimento de interpretação, os dados foram contaminados com ruídos aleatório Gaussiano cujo desvio padrão foi de $3 \%$. Isto equivale a um erro nas medidas de até $9 \%$.

$\mathrm{Na}$ modelagem direta foi utilizado para as medidas o arranjo dipolo-dipolo com espaçamento entre os eletrodos de $2 \mathrm{~m}$, a região de interesse foi discretizada em 20×76, totalizando 1520 elementos quadrados, em cada elemento a resistividade foi considerada constante.

No processo inverso, o modelo inicial foi tomado como um meio homogêneo com resistividade dada pela média das resistividades aparentes obtidas pela modelagem direta. $\mathrm{O}$ processo foi simulado em computador com processador core i5, com 4 Gb de memória RAM, onde rodou em 10 min. Bem menos que para dados de polarização induzida[3].

O modelo considerado foi um com três camadas, sendo a primeira e a segunda camadas são horizontais com resistividade de $64 \Omega m$ e $130 \Omega m$, respectivamente. Na terceira camada existe um contato vertical dividindo a mesma em duas, uma com resistividade de $270 \Omega m$ e a outra com $743 \Omega m$, conforme Figura 1.

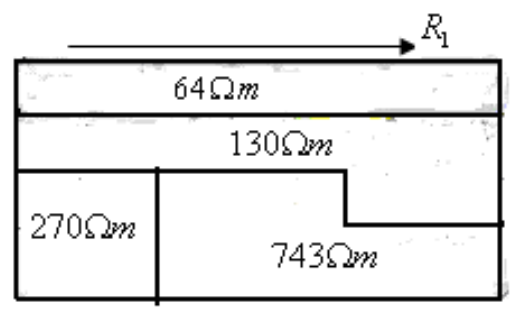

Figura 1- Desenho esquemático da perspectiva do terreno estudado cujo perfil das sondagens do subsolo na direção da seta $R_{1}$ com resistividades $64 \Omega m, 130 \Omega m, 270 \Omega m$ e $743 \Omega m$.

Foi detectada uma anomalia nos resultados da modelagem direta por meio da pseudo-seção, entretanto, não se observou a percepção clara da existência de um contato vertical (Figura 2).

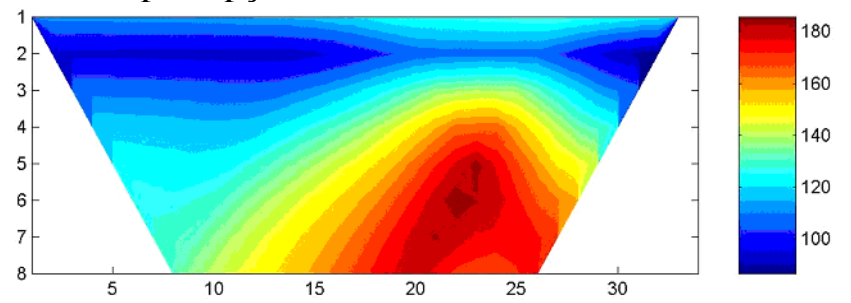

Figura 2- Modelagem direta 2D do solo com resistividades $64 \Omega m, 130 \Omega m, 270 \Omega m$ e $743 \Omega m$ na direção da seta $R_{1}$, obtido com o arranjo diplo-dipolo. A modelagem 2D evidenciou um contraste de resistividades na superfície detectando contato geológico vertical. 
Foi claramente detectado um contato vertical no resultado da inversão dos dados na imagem abaixo.

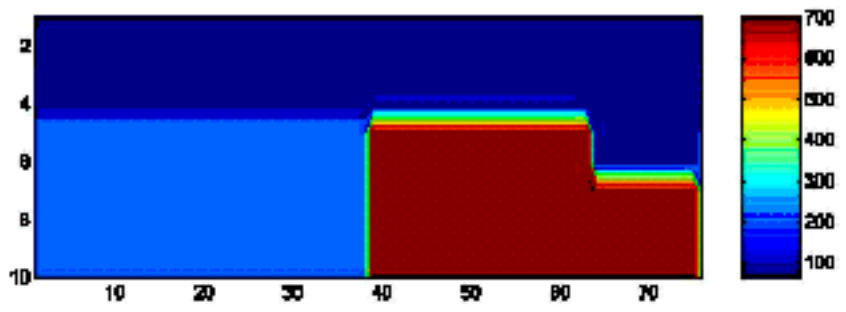

Figura 3- Modelo inverso.

Na Figura 3 observa-se uma camada bem resistente, limitada na base e no topo por porções menos resistentes. Essa variação pode ser relacionada ao elevado grau de heterogeneidade e/ou anisotropia estrutural deposicional e diagenética observada nos intervalos arenosos (Rostirolla et al. 2000, Trzaskos-Lipksi et al. 2003). Desta forma pode-se identificar as zonas fraturadas pela análise de deslocamentos ou perturbações nos valores de resistividade.

\section{Conclusões}

Os resultados obtidos mostram que o método de Gauss-Newton aplicado em inversão de dados de resistividade elétrica do solo mostrou um bom desempenho e com menor tempo computacional que na aplicação deste método em dados de polarização induzida. No modelo sintético simulado neste trabalho, foi possível detectar contatos verticais com bastante clareza.

\section{VII - Referencias}

[1] Maranhão, Celsa H. Melo, "Análise da Influencia da Heterogeneidade da Resistividade do Solo em Sistemas de Aterramentos", Tese de Doutorado, Programa de Pós-Graduação em Engenharia Elétrica, Centro Tecnológico, Universidade Federal do Pará, 2008.

[2] Maranhão, Celsa H. M.; Farias, Valcir J da C.; Rocha, Brígida R. P. , Modelagem Direta 2D de Terrenos com Heterogeneidade Lateral para um Sistema de Aterramento Elétrico, IEEE Latin America Transactions, vol. 6, no. 5, September 2008.

[3] Farias, V. J. da C., Rocha, B. R. P. da, Rocha, M. P. da C. da, Tavares, H. R. The use of fractal model to complex resistivity in the interpretation of induced polarization, Applied Mathematical Modelling, 37, 1347-1361, 2013.

[4] Farias, V. J. da C., Maranhão, C. H M., Rocha, B. R. P. da, Andrade, N. P. O. Induced polarization forward modelling using finite element method and fractal model. Applied Mathematical Modelling, 34, 1849-1860, 2010.

[5] Rostirolla, S. P.; Assine, M. I.; Fernandes, L. A.; Arthur, P. C. Reativação de paleolineamentos durante a evolução da Bacia do Paraná: o exemplo do Alto Estrutural de Quatiguá. Revista Brasileira de Geociências, São Paulo, v. 31, p. 639-648, 2000.

[6] V. J. da C. Farias, "Interpretação de Dados de Polarização Induzida usando o Modelo Fractal para Resistividades Complexas e Imagens Tomográficas”, Programa de Pós- 
Graduação em Engenharia Elétrica, Centro Tecnológico, Universidade Federal do Pará, Tese de Doutorado, p.151, 2004.

[7] Trzaskos-Lipski, B.; Rostirolla, S. P.; Vesely, F. F.; Bocardi, L.; Mancini, F.; Appi, C. J. Microtectônica e sua importância no estudo da circulação de fluidos em reservatórios fraturados do Grupo Itararé - Bacia do Paraná. In: Simpósio Nacional de Estudos Tectônicos - SNET, 9., 2003, Búzios. Anais...Rio de Janeiro: Sociedade Brasileira de Geologia, p. 361.

[8] Xia, J. J.; Habashy,T. M., Kong, J. A., 1994 - Profile Inversion in a Cylindrically Stratified Lossy Medium Radio Science, V 29, p. 1131 - 1141. 